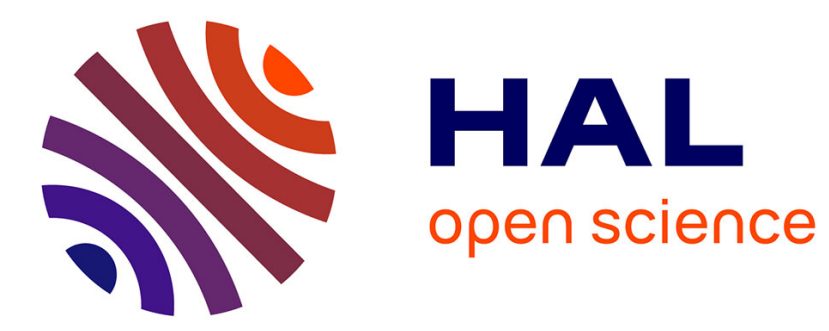

\title{
On-line Bicriteria Interval Scheduling
}

Fabien Baille, Evripidis Bampis, Christian Laforest, Nicolas Thibault

\section{To cite this version:}

Fabien Baille, Evripidis Bampis, Christian Laforest, Nicolas Thibault. On-line Bicriteria Interval Scheduling. Euro-Par 2005, 2005, Portugal. pp.312-322, 10.1007/11549468_36 . hal-00341367

\section{HAL Id: hal-00341367 https://hal.science/hal-00341367}

Submitted on 18 Jul 2009

HAL is a multi-disciplinary open access archive for the deposit and dissemination of scientific research documents, whether they are published or not. The documents may come from teaching and research institutions in France or abroad, or from public or private research centers.
L'archive ouverte pluridisciplinaire HAL, est destinée au dépôt et à la diffusion de documents scientifiques de niveau recherche, publiés ou non, émanant des établissements d'enseignement et de recherche français ou étrangers, des laboratoires publics ou privés. 


\title{
On-line Bicriteria Interval Scheduling
}

\author{
Fabien Baille, Evripidis Bampis, Christian Laforest, and Nicolas Thibault \\ LaMI, CNRS UMR 8042, Université d'Evry, \\ Tour Evry 2, 523, Place des Terrasses 91000 Evry, France \\ \{fbaille, bampis, laforest, nthibaul\}@lami.univ-evry.fr
}

\begin{abstract}
We consider the problem of scheduling a sequence of intervals revealed one by one in the order of their release dates on a set of $k$ identical machines. Each interval $i$ is associated with a processing time $p_{i}$ and a couple of arbitrary weights $\left(w_{i}^{A}, w_{i}^{B}\right)$ and may be scheduled on one of the $k$ identical machines or rejected. The objective is to determine a valid schedule maximizing the sum of the weights of the scheduled intervals for each coordinate. We first propose a generic on-line algorithm based on the combination of two monocriteria on-line algorithms and we prove that it gives rise to a couple of competitive ratios that are function of the competitive ratios of the monocriteria algorithms in the input. We apply this technique to the special case where $w_{i}^{A}=1$ and $w_{i}^{B}=p_{i}$ and as a corollary we obtain a couple of constant competitive ratios.
\end{abstract}

\section{Introduction}

We consider the problem of scheduling in an on-line context a set of $n$ intervals on $k$ identical machines. An interval $i$ is defined as a tuple of five positive real numbers $\left(r_{i}, p_{i}, d_{i}, w_{i}^{A}, w_{i}^{B}\right)$, where $r_{i}$ denotes the release date, $p_{i}$ the processing time, $d_{i}=r_{i}+p_{i}$ the deadline and $w_{i}^{A}$ and $w_{i}^{B}$ two arbitrary weights. We consider the following on-line context: Intervals arrive in increasing order of their release dates (i.e. $r_{1} \leq r_{2} \leq \cdots \leq r_{i} \leq \cdots$ ) and they are not known before their release dates. Let $\sigma=\sigma_{1}, \ldots, \sigma_{n}$ be the sequence of intervals in the input of the algorithm. For any two intervals $\sigma_{i}$ and $\sigma_{j}$, if $i<j$ then $r_{i} \leq r_{j}$. An interval can either be served, interrupted or rejected. An interval $i$ is said to be served or accepted if it is alloted exclusively and without interruption (preemption is not allowed) to one of the $k$ machines from date $r_{i}$ to date $d_{i}$. A schedule $O$ is valid if every served interval is scheduled at most once and if at each date every machine schedules at most one interval. There are two objective functions that we called the $W_{A}$, defined as the sum of the first-coordinate-weights $w_{i}^{A}$ of the accepted intervals, and the weight $W_{B}$, corresponding to the sum of the second-coordinate-weights $w_{i}^{B}$ of the accepted intervals in $O$. We search for a solution that simultaneously maximizes these two objectives. When the context is more general, we denote the weight of a schedule $O$ by $W(O)$. The particular weight function $w_{i}^{A}=1$ (resp. $w-i^{B}=p_{i}$ ) corresponds to the well known SIzE (resp. PROPORTIONAL WEIGHT) problems. 
Competitive ratio. In order to analyze the performance of an on-line algorithm, we use the notion of competitive ratio $[4,7]$. Let $\sigma_{1}, \cdots, \sigma_{n}$ be any on-line sequence. For every $i, 1 \leq i \leq n$, let $A\left(\sigma_{1}, \cdots, \sigma_{i}\right)$ be the schedule returned by the algorithm $A$ at step $i$, i.e. when the first $i$ intervals are revealed, and let $O_{i}^{*}$ be an optimal schedule for some metric $C$. Then $A$ is said to be $\rho$-competitive for the metric $C$ if, for all $i(1 \leq i \leq n)$, the following inequality holds:

$$
\rho \bar{C}\left(A\left(\sigma_{1}, \cdots, \sigma_{n}\right)\right) \geq C\left(O_{i}^{*}\right)
$$

For our bicriteria problem, an algorithm $A$ is said to be $(\rho, \mu)$-competitive if it is simultaneously $\rho$-competitive for the weight $W_{A}$ and $\mu$-competitive for the weight $W_{B}$.

Previous works. To the best of our knowledge, this is the first work considering the simultaneous maximization of two different weight functions in an on-line context. Nevertheless, the off-line version of the bicriteria problem has been treated in [2] where a $\left(\frac{k}{r}, \frac{k}{k-r}\right)$-approximation algorithm $(1 \leq r<k)$ has been proposed. On the contrary, the monocriteria problems have been extensively studied for both the off-line and the on-line versions. In particular, the off-line versions are polynomial (see Faigle and Nawijn [6] for the SIZE and Carlisle and Lloyd [5] or Arkin and Silverberg [1] for the WEIGHT problems). In the on-line context, the algorithm $G O L$ of Faigle and Nawijn [6] is optimal for the SIZE problem. For the WEIGHT problem, there is a series of works going from the paper of Woeginger, in [8], who proposed a 4-competitive algorithm for the PROPORTIONAL WEIGHTS problem in a single machine system, to the paper of Bar-Noy et al. [3] who proposed the $L R$ algorithm which is $\frac{2}{1-2 \delta}$-competitive for PROPORTIONAL WEIGHTS in a different model than ours (instead of $k$ machine, they consider a continuous channel where an interval requires less than a portion $\delta$ of the total channel).

Outline of the paper. In Section 2, we describe a generic on-line algorithm for the simultaneous maximization of two weight functions $W_{A}$ and $W_{B}$. We prove that it is a $\left(\frac{k}{r} \rho, \frac{k}{k-r} \mu\right)$-competitive algorithm, for $1 \leq r \leq k$, where $\rho$ and $\mu$ are the competitive ratios of the corresponding monocriteria problems. However, up to our knowledge no on-line algorithm is available for the general WEIGHT problem. So, we focus, in Section 3, on the special case of the size and proportional weights metrics. We first study the monocriteria competitiveness of $L R^{k}$ (the adaptation of $L R$ in our model of $k$ machines) for the proportional weight criterion. This result is then combined with the competitive ratio of $G O L^{k}$ (the algorithm $G O L$ running on $k$ machines) for the size criterion along with the one of our generic method, allowing us to prove a couple of constant competitive ratios for this bicriteria case.

\section{Our Generic Bicriteria Algorithm.}

In this section, we describe our generic bicriteria on-line algorithm. It uses as subroutines two on-line monocriteria algorithms having the following structure. 
Structure of the monocriteria algorithms. At the release date $r_{i}$ of a new interval $\sigma_{i}$, any on-line monocriterion algorithm can be split into two main stages. In the first one, called the interrupting stage, a set of already scheduled intervals are selected to be interrupted at time $r_{i}$. This set can potentially be empty meaning that no interval is interrupted when the algorithm considers $\sigma_{i}$. The second stage is the scheduling stage. Here, the algorithm can either reject the interval $\sigma_{i}$ or schedule it on one of the available machines.

The rough idea of our generic algorithm is the following: it simulates the execution of two algorithms, say $A$ for the maximization of the weight $W_{A}$ and $B$ for the maximization of the weight $W_{B}$ on $r$ and $k-r$ machines, respectively. By doing this, it builds its own interrupting (resp. scheduling) stage from the corresponding interrupting (resp. scheduling) stage of the input algorithms.

\subsection{The algorithm $A B^{k}$}

We consider the $i$-th step of an arbitrary algorithm for the WEIGHT problem, i.e. the step at which interval $\sigma_{i}$ is released. For any algorithm $A L G$ and for every execution step $i$ of this algorithm, let $\mathcal{O}_{i_{1}}(A L G)$ (resp. $\left.\mathcal{O}_{i_{2}}(A L G)\right)$ be the schedule given by $A L G$ after the execution of its interrupting (resp. scheduling) stage of step $i$.

Given two algorithms $A$ for the maximization of the weight $W_{A}$ and $B$ for the weight $W_{B}$, our generic algorithm $A B^{k}$ is constructed as follows: $A B^{k}$ builds the final schedule by combining the schedules returned by algorithms $A$ and $B$ when applied on $r$ machines and $k-r$ machines, respectively. For the ease of presentation, we denote by $A^{r}$ (resp. $B^{k-r}$ ) the algorithm $A$ (resp. $B$ ) when applied on $r$ (resp. $k-r$ ) machines. We also call real (resp. virtual) the machines involved in the algorithm $A B^{k}$ (resp. $A^{r}$ and $B^{k-r}$ ).

For every execution step $i$ of $A B^{k}$, let $\mathcal{R}_{i_{1}}\left(A B^{k}\right)$ (resp. $\mathcal{R}_{i_{2}}\left(A B^{k}\right)$ ) be the set of scheduled intervals after the interrupting (resp. scheduling) stage of step $i$ on the real machines associated to $A B^{k}$.

For every execution step $i$ of the algorithm $A^{r}$ (resp. $B^{k-r}$ ), let $\mathcal{V}_{i_{1}}\left(A^{r}\right)$ (resp. $\mathcal{V}_{i_{1}}\left(B^{k-r}\right)$ ) be the set of scheduled intervals after the interrupting stage of step $i$ on the $r$ (resp. $k-r$ ) virtual machines associated to $A^{r}$ (resp. $B^{k-r}$ ), and let $\mathcal{V}_{i_{2}}\left(A^{r}\right)$ (resp. $\mathcal{V}_{i_{2}}\left(B^{k-r}\right)$ ) be the set of scheduled intervals after the scheduling stage of step $i$ on the $r$ (resp. the $k-r$ ) virtual machines associated to $A^{r}$ (resp. $\left.B^{k-r}\right)$.

\section{Algorithm $A B^{k}$}

Input: $k$ identical machines and an on-line sequence of intervals $\sigma_{1}, \ldots, \sigma_{n}$.

Output: After each step $i(1 \leq i \leq n)$, a valid schedule $\mathcal{O}_{i_{2}}\left(A B^{k}\right)$ involving a subset of $\sigma_{1}, \ldots, \sigma_{i}$ on $k$ real machines.

Step 0: $\mathcal{V}_{0_{2}}\left(A^{r}\right)=\mathcal{V}_{0_{2}}\left(B^{k-r}\right)=\mathcal{R}_{0_{2}}\left(A B^{k}\right)=\emptyset$

Step $i$ (date $\left.r_{i}\right)$ :

1. The interrupting stage of $A B^{k}$ :

(a) Execute the interrupting stage of $A^{r}$ (resp. $B^{k-r}$ ) on the $r$ (resp. $k-r$ ) virtual machines associated to $A^{r}$ (resp. $B^{k-r}$ ) by submitting 
the new interval $\sigma_{i}$ to $A^{r}$ (resp. $B^{k-r}$ ). Note that the set of intervals scheduled and not interrupted by $A^{r}$ (resp. $B^{k-r}$ ) is now $\mathcal{V}_{i_{1}}\left(A^{r}\right)$ $\left(\right.$ resp. $\left.\mathcal{V}_{i_{1}}\left(B^{k-r}\right)\right)$.

(b) Execute the interrupting stage of $A B^{k}$ on the $k$ real machines associated to $A B^{k}$ by interrupting the intervals of $\mathcal{R}_{(i-1)_{2}}\left(A B^{k}\right)$ such that after this interruption we get:

2. The scheduling stage of $A B^{k}$ :

$$
\mathcal{R}_{i_{1}}\left(A B^{k}\right)=\mathcal{V}_{i_{1}}\left(A^{r}\right) \cup \mathcal{V}_{i_{1}}\left(B^{k-r}\right)
$$

(a) Execute the scheduling stage of $A^{r}$ (resp. $B^{k-r}$ ) on the $r$ (resp. $k-r$ ) virtual machines associated to $A^{r}$ (resp. $B^{k-r}$ ) by serving or rejecting the new interval $\sigma_{i}$.

(b) Execute the scheduling stage of $A B^{k}$ on the $k$ real machines associated to $A B^{k}$ by switching to the appropriate case:

i. If $A^{r}$ and $B^{k-r}$ reject $\sigma_{i}$, then $A B^{k}$ does not schedule (rejects) $\sigma_{i}$. Thus, we have:

$$
\mathcal{R}_{i_{2}}\left(A B^{k}\right)=\mathcal{R}_{i_{1}}\left(A B^{k}\right)
$$

ii. If $A^{r}$ or $B^{k-r}$ serves $\sigma_{i}$ (including the case in which both $A^{r}$ and $B^{k-r}$ serve $\sigma_{i}$ ), then $A B^{k}$ schedules $\sigma_{i}$ on any free real machine at time $r_{i}$. Thus, we have:

$$
\mathcal{R}_{i_{2}}\left(A B^{k}\right)=\mathcal{R}_{i_{1}}\left(A B^{k}\right) \cup\left\{\sigma_{i}\right\}
$$

\subsection{Competitiveness of $A B^{k}$}

Here, we analyze the competitiveness of $A B^{k}$. We start with the following lemma which states that $A B^{k}$ returns a valid schedule and executes the same set of intervals as the union of $A^{r}$ and $B^{k-r}$.

Lemma 1 For every step $i$ of the algorithm $A B^{k}$, the schedule $\mathcal{O}_{i_{2}}\left(A B^{k}\right)$ is valid and we have:

$$
\mathcal{R}_{i_{2}}\left(A B^{k}\right)=\mathcal{V}_{i_{2}}\left(A^{r}\right) \cup \mathcal{V}_{i_{2}}\left(B^{k-r}\right)
$$

Proof. We prove this lemma by induction on the execution steps $i$ of $A B^{k}$.

The basic case (step 0): By definition $\mathcal{V}_{0_{2}}\left(A^{r}\right)=\mathcal{V}_{0_{2}}\left(B^{k-r}\right)=\mathcal{R}_{0_{2}}\left(A B^{k}\right)=\emptyset$ and thus, $\mathcal{O}_{i_{2}}\left(A B^{k}\right)$ is valid and of course $\mathcal{R}_{0_{2}}\left(A B^{k}\right)=\mathcal{V}_{0_{2}}\left(A^{r}\right) \cup \mathcal{V}_{0_{2}}\left(B^{k-r}\right)$. The main case (step i): Let us assume that $\mathcal{O}_{(i-1)_{2}}\left(A B^{k}\right)$ is valid and that $\mathcal{R}_{(i-1)_{2}}\left(A B^{k}\right)=\mathcal{V}_{(i-1)_{2}}\left(A^{r}\right) \cup \mathcal{V}_{(i-1)_{2}}\left(B^{k-r}\right)$ (assumption of induction).

1. The interrupting stage: We first need to prove that:

$\mathcal{R}_{i_{1}}\left(A B^{k}\right)=\mathcal{V}_{i_{1}}\left(A^{r}\right) \cup \mathcal{V}_{i_{1}}\left(B^{k-r}\right)$ and that $\mathcal{O}_{i_{1}}\left(A B^{k}\right)$ is valid.

(a) By definition, $A B^{k}$ interrupts a subset of intervals of $\mathcal{R}_{(i-1)_{2}}\left(A B^{k}\right)$ in such a way that:

$$
\mathcal{R}_{i_{1}}\left(A B^{k}\right)=\mathcal{V}_{i_{1}}\left(A^{r}\right) \cup \mathcal{V}_{i_{1}}\left(B^{k-r}\right)
$$

We have to show that there is always a subset of $\mathcal{R}_{(i-1)_{2}}\left(A B^{k}\right)$ that can be removed such that the above equality is possible.

Since $\mathcal{V}_{i_{1}}\left(A^{r}\right) \subseteq \mathcal{V}_{(i-1)_{2}}\left(A^{r}\right), \mathcal{V}_{i_{1}}\left(B^{k-r}\right) \subseteq \mathcal{V}_{(i-1)_{2}}\left(B^{k-r}\right)$ and given that $\mathcal{R}_{(i-1)_{2}}\left(A B^{k}\right)=\mathcal{V}_{(i-1)_{2}}\left(A^{r}\right) \cup \mathcal{V}_{(i-1)_{2}}\left(B^{k-r}\right)$ (by the induction hypothesis), we have $\mathcal{V}_{i_{1}}\left(A^{r}\right) \cup \mathcal{V}_{i_{1}}\left(B^{k-r}\right) \subseteq \mathcal{R}_{(i-1)_{2}}\left(A B^{k}\right)$. 
(b) By definition, $A B^{k}$ interrupts only intervals scheduled in $\mathcal{O}_{(i-1)_{2}}\left(A B^{k}\right)$, and by the induction hypothesis, $\mathcal{O}_{(i-1)_{2}}\left(A B^{k}\right)$ is valid. Thus, $\mathcal{O}_{i_{1}}\left(A B^{k}\right)$ is clearly valid.

2. The scheduling stage: Now, we have to prove that:

$\mathcal{R}_{i_{2}}\left(A B^{k}\right)=\mathcal{V}_{i_{2}}\left(A^{r}\right) \cup \mathcal{V}_{i_{2}}\left(B^{k-r}\right)$ and that $\mathcal{O}_{i_{2}}\left(A B^{k}\right)$ is valid. By the definition of $A B^{k}$, several cases may occur:

(a) If $A^{r}$ and $B^{k-r}$ reject $\sigma_{i}$, then $A B^{k}$ does not schedule $\sigma_{i}$ and we have:

i. $\mathcal{R}_{i_{2}}\left(A B^{k}\right)=\mathcal{R}_{i_{1}}\left(A B^{k}\right)$ (by the definition of $A B^{k}$ )

$$
\begin{aligned}
& =\mathcal{V}_{i_{1}}\left(A^{r}\right) \cup \mathcal{V}_{i_{1}}\left(B^{k-r}\right)(\text { by }(1)) \\
& =\mathcal{V}_{i_{2}}\left(A^{r}\right) \cup \mathcal{V}_{i_{2}}\left(B^{k-r}\right)
\end{aligned}
$$

(since $A^{r}$ and $B^{k-r}$ reject $\sigma_{i}$, we have:

$\mathcal{V}_{i_{1}}\left(A^{r}\right)=\mathcal{V}_{i_{2}}\left(A^{r}\right)$ and $\left.\mathcal{V}_{i_{1}}\left(B^{k-r}\right)=\mathcal{V}_{i_{2}}\left(B^{k-r}\right)\right)$

ii. $\mathcal{O}_{i_{2}}\left(A B^{k}\right)=\mathcal{O}_{i_{1}}\left(A B^{k}\right)$. Thus $\mathcal{O}_{i_{2}}\left(A B^{k}\right)$ is valid (because in item $1 \mathrm{~b}$ of this proof, we have already seen that $\mathcal{O}_{i_{1}}\left(A B^{k}\right)$ is valid).

(b) If $A^{r}$ (resp. $B^{k-r}$ ) serves $\sigma_{i}$ and $B^{k-r}$ (resp. $A^{r}$ ) rejects $\sigma_{i}$, then $A B^{k}$ schedules $\sigma_{i}$ on any free real machine at time $r_{i}$. We have:

i. $\mathcal{R}_{i_{2}}\left(A B^{k}\right)=\mathcal{R}_{i_{1}}\left(A B^{k}\right) \cup\left\{\sigma_{i}\right\}$ (by the definition of $A B^{k}$ )

$$
\begin{aligned}
& =\mathcal{V}_{i_{1}}\left(A^{r}\right) \cup \mathcal{V}_{i_{1}}\left(B^{k-r}\right) \cup\left\{\sigma_{i}\right\} \text { (by (1)) } \\
& =\mathcal{V}_{i_{2}}\left(A^{r}\right) \cup \mathcal{V}_{i_{2}}\left(B^{k-r}\right)
\end{aligned}
$$

(since $A^{r}$ (resp. $B^{k-r}$ ) serves $\sigma_{i}$ and $B^{k-r}$ (resp. $A^{r}$ ) rejects $\sigma_{i}$, we have: $\mathcal{V}_{i_{2}}\left(A^{r}\right)=\mathcal{V}_{i_{1}}\left(A^{r}\right) \cup\left\{\sigma_{i}\right\}\left(\operatorname{resp} . \mathcal{V}_{i_{2}}\left(B^{k-r}\right)=\mathcal{V}_{i_{1}}\left(B^{k-r}\right) \cup\left\{\sigma_{i}\right\}\right)$ and $\left.\mathcal{V}_{i_{2}}\left(B^{k-r}\right)=\mathcal{V}_{i_{1}}\left(B^{k-r}\right)\left(\operatorname{resp} . \mathcal{V}_{i_{2}}\left(A^{r}\right)=\mathcal{V}_{i_{1}}\left(A^{r}\right)\right)\right)$

ii. Since $\mathcal{O}_{i_{1}}\left(A B^{k}\right)$ is a valid schedule (by the item $1 \mathrm{~b}$ of this proof) and $\mathcal{O}_{i_{2}}\left(A B^{k}\right)$ is built by adding $\sigma_{i}$ to $\mathcal{O}_{i_{1}}\left(A B^{k}\right)$ only once, the only reason for which $\mathcal{O}_{i_{2}}\left(A B^{k}\right)$ could not be valid would be because $\sigma_{i}$ is scheduled by $A B^{k}$ at time $r_{i}$ whereas there is no free machine at time $r_{i}$, i.e. because there is at least $k+1$ intervals of $\mathcal{R}_{i_{2}}\left(A B^{k}\right)$ scheduled at time $r_{i}$ by $A B^{k}$. Let us prove that this is impossible. Indeed, since $A^{r}$ and $B^{k-r}$ build at each time valid schedules, there are at most $r+k-r=k$ intervals of $\mathcal{V}_{i_{2}}\left(A^{r}\right) \cup \mathcal{V}_{i_{2}}\left(B^{k-r}\right)$ scheduled at time $r_{i}$ by $A^{r}$ and $B^{k-r}$, and thus, there are at most $k$ intervals of $\mathcal{R}_{i_{2}}\left(A B^{k}\right)$ scheduled at time $r_{i}$ by $A B^{k}$ (because we have just proved above that $\left.\mathcal{R}_{i_{2}}\left(A B^{k}\right)=\mathcal{V}_{i_{2}}\left(A^{r}\right) \cup \mathcal{V}_{i_{2}}\left(B^{k-r}\right)\right)$. Thus, $\mathcal{O}_{i_{2}}\left(A B^{k}\right)$ is a valid schedule.

(c) If $A^{r}$ and $B^{k-r}$ serve $\sigma_{i}$, then $A B^{k}$ schedules $\sigma_{i}$ on any idle machine at time $r_{i}$ and we get:

i. $\mathcal{R}_{i_{2}}\left(A B^{k}\right)=\mathcal{R}_{i_{1}}\left(A B^{k}\right) \cup\left\{\sigma_{i}\right\}$ (by the definition of $A B^{k}$ )

$$
\begin{aligned}
& \left.=\mathcal{V}_{i_{1}}\left(A^{r}\right) \cup \mathcal{V}_{i_{1}}\left(B^{k-r}\right) \cup\left\{\sigma_{i}\right\} \text { (by } 1\right) \\
& =\mathcal{V}_{i_{2}}\left(A^{r}\right) \cup \mathcal{V}_{i_{2}}\left(B^{k-r}\right)
\end{aligned}
$$

(since $A^{r}$ and $B^{k-r}$ serve $\sigma_{i}$, we have $\mathcal{V}_{i_{2}}\left(A^{r}\right)=\mathcal{V}_{i_{1}}\left(A^{r}\right) \cup\left\{\sigma_{i}\right\}$ and $\left.\mathcal{V}_{i_{2}}\left(B^{k-r}\right)=\mathcal{V}_{i_{1}}\left(B^{k-r}\right) \cup\left\{\sigma_{i}\right\}\right)$

ii. We prove that $\mathcal{O}_{i_{2}}\left(A B^{k}\right)$ is valid in the same way as before.

A direct consequence of Lemma 1 is that $A B^{k}$ is better than $A^{r}$ (resp. $B^{k-r}$ ) for the weight function that $A^{r}$ (resp. $B^{k-r}$ ) maximizes. 
Corollary 1 Let $W_{A}$ and $W_{B}$ be two arbitrary weight functions. For every input sequence $\sigma_{1}, \ldots, \sigma_{n}$ and for each step $i(1 \leq i \leq n)$ of the algorithm $A B^{k}$, we have:

$$
W_{A}\left(\mathcal{V}_{i_{2}}\left(A^{r}\right)\right) \leq W_{A}\left(\mathcal{R}_{i_{2}}\left(A B^{k}\right)\right) \text { and } W_{B}\left(\mathcal{V}_{i_{2}}\left(B^{k-r}\right)\right) \leq W_{B}\left(\mathcal{R}_{i_{2}}\left(A B^{k}\right)\right)
$$

Proof. By Lemma 1, for every step $i$ of the algorithm $A B^{k}$, we have $\mathcal{R}_{i_{2}}\left(A B^{k}\right)=\mathcal{V}_{i_{2}}\left(A^{r}\right) \cup \mathcal{V}_{i_{2}}\left(B^{k-r}\right)$ and thus Corollary 1 is valid.

In the following lemma, we show that for any type of weight function $W$, if an on-line algorithm $A$ is $\rho$-competitive for $W$ on $k$ machines, then $A^{r}$ consisting in applying $A$ on $r \leq k$ machines is $\left(\frac{k}{r} \rho\right)$-competitive compared to an optimal schedule on $k$ machines.

Lemma 2 Let $\sigma_{1}, \cdots, \sigma_{n}$ be any on-line sequence of intervals. Let $A$ an on-line algorithm with competitiveness $\rho$ on $r$ machines $(r \leq k)$ and $O_{k}^{*}$ (resp. $\left.O_{r}^{*}\right)$ be an optimal schedule of $\sigma_{1}, \cdots, \sigma_{n}$ for the weight function $W$ on $k$ (resp. $r$ ) machines and $O_{r}$ be the schedule returned by $A_{r}$ on $\sigma_{1}, \cdots, \sigma_{n}$ on $r$ machines. Then,

$$
W\left(O_{k}^{*}\right) \leq \frac{k}{r} \rho W\left(O_{r}\right)
$$

Proof. Since $A$ is $\rho$-competitive, we have by definition $W\left(O_{r}^{*}\right) \leq \rho W\left(O_{r}\right)$. If we multiply both sides of this inequation by $\frac{k}{r}$, we get $\frac{k}{r} W\left(O_{r}^{*}\right) \leq \frac{k}{r} \rho W\left(O_{r}\right)$.

Let $O_{1}$ be the schedule composed of the first $r$ machines of $O_{k}^{*}$ in the decreasing order of their weights. Since $O_{1}$ is a $r$-machine schedule, its weight is at most $W\left(O_{r}^{*}\right)$. We thus have:

$$
\frac{k}{r} W\left(O_{1}\right) \leq \frac{k}{r} W\left(O_{r}^{*}\right) \leq \frac{k}{r} \rho W\left(O_{r}\right)
$$

Since $O_{1}$ is a schedule on $r$ machines executing the intervals scheduled in the $r$ machines generating the maximum weight in $O_{k}^{*}$, the average weight per machine in $O_{1}$ is greater than the average weight per machine in $O_{k}^{*}$. Thus, we have: $\frac{W\left(O_{k}^{*}\right)}{k} \leq \frac{W\left(O_{1}\right)}{r}$. Combining this result with (2), we get: $W\left(O_{k}^{*}\right) \leq \frac{k}{r} \rho W\left(O_{r}\right)$

We finish with the bi-competitiveness analysis of $A B^{k}$ for two general weights.

Theorem 1 Let $\sigma_{1}, \cdots, \sigma_{n}$ be any on-line sequence of intervals. If $A^{r}$ is a $\rho$ competitive algorithm for the weight function $W_{A}$ on $r$ machines and $B^{k-r}$ is a $\mu$-competitive algorithm for the weight function $W_{B}$ on $k-r$ machines, then the algorithm $A B^{k}$ using $A^{r}$ and $B^{k-r}$ as subroutines is $\left(\frac{k}{r} \rho, \frac{k}{k-r} \mu\right)$-competitive.

Proof. Let $O_{k}^{*}(A)$ be an optimal schedule of $\sigma_{1}, \ldots, \sigma_{n}$ on $k$ machines for the weight function $W_{A}$ and $O_{k}^{*}(B)$ be an optimal schedule of $\sigma_{1}, \ldots, \sigma_{n}$ on $k$ machines for the weight function $W_{B}$. By Lemma 2, we have:

$$
W_{A}\left(O_{k}^{*}(A)\right) \leq \frac{k}{r} \rho W_{A}\left(\mathcal{V}_{i_{2}}\left(A^{r}\right)\right) \text { and } W_{B}\left(O_{k}^{*}(B)\right) \leq \frac{k}{k-r} \mu W_{B}\left(\mathcal{V}_{i_{2}}\left(B^{k-r}\right)\right)
$$

Moreover, using Corollary 1 , we have:

$$
W_{A}\left(O_{k}^{*}(A)\right) \leq \frac{k}{r} \rho W_{A}\left(\mathcal{R}_{i_{2}}\left(A B^{k}\right)\right) \text { and } W_{B}\left(O_{k}^{*}(B)\right) \leq \frac{k}{k-r} \mu W_{B}\left(\mathcal{R}_{i_{2}}\left(A B^{k}\right)\right)
$$

Thus $A B^{k}$ is $\left(\frac{k}{r} \rho, \frac{k}{k-r} \mu\right)$-competitive. 


\section{Application to the SIZE and the PROPORTIONAL WEIGHT}

Given that no on-line algorithm is known for general weight functions, we focus in this section on the particular case where $w_{i}^{A}=1$ and $w_{i}^{B}=p_{i}$ for every $i=1, \ldots, n$, i.e. for the size and proportional weights metrics. We first show that the optimal on-line algorithm GOL of Faigle and Nawijn [6] can be described following the two-stages structure presented in the previous section. We also present in this form the on-line algorithm $L R^{k}$ of Bar-Noy et al. [3]. Recall that $G O L^{k}$ is optimal for the SIZE problem while $L R$ deals with proportional weights (but for a different model than the one adopted here). Thus, before using these two algorithms as input for our generic method we need to evaluate the competitive ratio of the algorithm for our model.

Here is a description of the algorithm $G O L^{k},[6]$. It is split into an interrupting stage and a scheduling stage.

\section{Algorithm $G O L^{k}[6]$}

At the arrival of interval $\sigma_{i}$ do:

Interrupting stage: If there are $k$ served intervals intersecting the date $r_{i}$, let $\sigma_{\max }$ be the one with the maximum deadline.

If $\sigma_{\max }$ does not exist (there is a free machine), do not interrupt any interval.

If $d_{\max } \geq d_{i}$ then interrupt $\sigma_{\max }$.

If $d_{\max }<d_{i}$ then do not interrupt any interval.

Scheduling stage: If an interval has been interrupted (a machine became idle) or if there is a free machine, then schedule $\sigma_{i}$ on any free machine. Else, reject $\sigma_{i}$.

We now adapt the algorithm $L R$. In [3], $L R$ is described as an algorithm running on a continuous channel, where each interval requires a portion (not necessarily contiguous) of this channel. In our model, instead of a continuous channel, we consider $k$ machines, and each interval requires exactly one machine. That is why we give the description of $L R^{k}$ (the adaptation of $L R$ on a discrete model of $k \geq 3$ machines) and the proof of its $\frac{2}{1-\frac{2}{k-r}}$-competitiveness. Note that Lemma 3 and Theorem 2 are just adaptations of the proof of competitiveness of $L R$ coming from [3] to our model.

\section{Algorithm $L R^{k}$ (adaptation of [3])}

We define $F_{t}$ as the set of scheduled intervals containing date $t$.

When $\sigma_{i}$ is revealed do:

Interrupting stage:

- If $\left|F_{r_{i}}\right|<k$, then do not interrupt any interval

- If $\left|F_{r_{i}}\right|=k$, then:

1. Sort the $k+1$ intervals of $F_{r_{i}} \cup\left\{\sigma_{i}\right\}$ by increasing order of release dates, if several intervals have the same release date, order them in the decreasing order of their deadlines and let $L$ be the set of the $\left\lceil\frac{k}{2}\right\rceil$ first intervals.

2. Sort the $k+1$ intervals of $F_{r_{i}} \cup\left\{\sigma_{i}\right\}$ by decreasing order of deadlines (ties are broken arbitrarily) and let $R$ be the set of the $\left\lfloor\frac{k}{2}\right\rfloor$ first intervals. 
If $\sigma_{i} \in L \cup R$ then interrupt any interval $\sigma_{j}$ of $F_{r_{i}}-L \cup R$.

Else do not interrupt any interval.

Scheduling stage:

- If $\left|F_{r_{i}}\right|<k$ then schedule $\sigma_{i}$ on any free machine.

- If $\left|F_{r_{i}}\right|=k$, then:

* If $\sigma_{i} \in L \cup R$ then schedule $\sigma_{i}$ on the machine where $\sigma_{j}$ was interrupted.

* If $\sigma_{i} \notin L \cup R$ then reject $\sigma_{i}$.

We now describe the notations needed by the proof of the competitive ratio of $L R^{k}$. Let $O=L R^{k}\left(\sigma_{1}, \cdots, \sigma_{i}\right)$ be the schedule on $k$ machines returned by $L R^{k}$ on $\sigma_{1}, \cdots, \sigma_{i}$. Let $T_{i}^{t}$ be the size of intervals of $O$ containing the date $t$. Let $F_{i}^{t}$ be the number of intervals of $\left\{\sigma_{1}, \cdots, \sigma_{i}\right\}$ containing the date $t$.

Lemma 3 Using the above notations, the schedule returned by $L R^{k}$ satisfies:

$$
\forall i, \forall t, T_{i}^{t} \geq \min \left\{F_{i}^{t}, \frac{k}{2}-1\right\}
$$

Proof. We proceed by induction on $i$. For $i=1, \forall t \in\left[r_{1}, d_{1}\right)$, we have:

$T_{1}^{t}=F_{1}^{t}=1$ and $\forall t \notin\left[r_{1}, d_{1}\right), T_{1}^{t}=F_{1}^{t}=0$.

Suppose $i>1$. According to the algorithm, two cases may occur:

1. $\left|F_{r_{i}}\right|<k$. In this case, $\sigma_{i}$ is scheduled by $L R^{k}$ and no interval is interrupted.

- If $t \notin\left[r_{i}, d_{i}\right)$, then the number of scheduled intervals which contain the date $t$ at step $i$ is the same as at step $i-1$. Thus, we have $T_{i}^{t}=T_{i-1}^{t}$. Moreover, since $t \notin\left[r_{i}, d_{i}\right)$, we have also $F_{i}^{t}=F_{i-1}^{t}$. So, by replacing $T_{i-1}^{t}$ by $T_{i}^{t}$ and $F_{i-1}^{t}$ by $F_{i}^{t}$ in the induction hypothesis, this particular case is checked.

- If $t \in\left[r_{i}, d_{i}\right)$, then since $\sigma_{i}$ has been scheduled, we have: $T_{i}^{t}=T_{i-1}^{t}+1$. By the induction hypothesis, we can rewrite this equation:

$$
T_{i}^{t} \geq 1+\min \left\{F_{i-1}^{t}, \frac{k}{2}-1\right\}
$$

If $\min \left\{F_{i-1}^{t}, \frac{k}{2}-1\right\}=\frac{k}{2}-1$, then (3) becomes:

$T_{i}^{t} \geq 1+\frac{k}{2}-1=\frac{k}{2}>\frac{k}{2}-1 \geq \min \left\{F_{i}^{t}, \frac{k}{2}-1\right\}$.

If $\min \left\{F_{i-1}^{t}, \frac{k}{2}-1\right\}=F_{i-1}^{t}$, then (3) becomes: $T_{i}^{t} \geq 1+F_{i-1}^{t}$. But since $t \in\left[r_{i}, d_{i}\right)$, we have $F_{i}^{t}=F_{i-1}^{t}+1$. Thus, we have:

$T_{i}^{t} \geq F_{i}^{t}-1+1=F_{i}^{t} \geq \min \left\{F_{i}^{t}, \frac{k}{2}-1\right\}$.

2. $\left|F_{r_{i}}\right|=\bar{k}$. In this case, three sub-cases may occur:

- If $\sigma_{i} \notin L$ and $\sigma_{i} \notin R$. This means that $\sigma_{i}$ is rejected by $L R^{k}$.

- If $t \notin\left[r_{i}, d_{i}\right)$ then $T_{i}^{t}=T_{i-1}^{t}$ and $F_{i}^{t}=F_{i-1}^{t}$. By replacing $T_{i-1}^{t}$ by $T_{i}^{t}$ and $F_{i-1}^{t}$ by $F_{i}^{t}$ in the induction hypothesis, this particular case is checked.

- If $t \in\left[r_{i}, d_{i}\right)$, since $\sigma_{i} \notin L \cup R$, there are always at least $\left\lfloor\frac{k}{2}\right\rfloor$ intervals containing $t$ in $O$. Thus, $T_{i}^{t} \geq\left\lfloor\frac{k}{2}\right\rfloor \geq \min \left\{F_{i}^{t}, \frac{k}{2}-1\right\}$.

- If $\sigma_{i} \in R$ (including the case where $\sigma_{i}$ is also in $L$ ). This means that $\sigma_{i}$ is accepted by $L R^{k}$ and $\sigma_{j}$ is rejected. Then, since $\sigma_{j}$ is revealed before $\sigma_{i}$, we have $r_{j} \leq r_{i}$. Furthermore, we have $d_{j} \leq d_{i}$ otherwise, we would have $\sigma_{j} \in R$, contradicting the fact that $\sigma_{j}$ is interrupted. We have then these cases: 
- For all $t \notin\left[r_{j}, d_{i}\right)$, we have $F_{i}^{t}=F_{i-1}^{t}$ and $T_{i}^{t}=T_{i-1}^{t}$. Thus, by replacing $T_{i-1}^{t}$ by $T_{i}^{t}$ and $F_{i-1}^{t}$ by $F_{i}^{t}$ in the induction hypothesis, this particular case is checked.

- For all $t \in\left[r_{j}, r_{i}\right)$, since $\sigma_{j} \notin L$, there are at least $\left\lceil\frac{k}{2}\right\rceil$ intervals containing the date $t$. Thus, we have: $T_{i}^{t} \geq\left\lceil\frac{k}{2}\right\rceil>\min \left\{F_{i}^{t}, \frac{k}{2}-1\right\}$.

- For all $t \in\left[r_{i}, d_{j}\right)$, we have $T_{i}^{t}=T_{i-1}^{t}$ because $\sigma_{j}$ is deleted but $\sigma_{i}$ is added. Since $\sigma_{j} \notin R$, there are at least $\left\lfloor\frac{k}{2}\right\rfloor$ intervals containing date $t$. Thus, we have $T_{i}^{t} \geq\left\lfloor\frac{k}{2}\right\rfloor \geq \min \left\{F_{i}^{t}, \frac{k}{2}-1\right\}$.

- For all $t \in\left[d_{j}, d_{i}\right)$, since $\sigma_{i}$ occupies a machine that was free at step $i-1$ of the algorithm, we have: $T_{i}^{t}=T_{i-1}^{t}+1$. By the induction hypothesis, we can rewrite this equation:

$$
T_{i}^{t} \geq 1+\min \left\{F_{i-1}^{t}, \frac{k}{2}-1\right\}
$$

If $\min \left\{F_{i-1}^{t}, \frac{k}{2}-1\right\}=\frac{k}{2}-1$, then (4) becomes:

$T_{i}^{t} \geq 1+\frac{k}{2}-1=\frac{k}{2}>\frac{k}{2}-1 \geq \min \left\{F_{i}^{t}, \frac{k}{2}-1\right\}$.

If $\min \left\{F_{i-1}^{t}, \frac{k}{2}-1\right\}=F_{i-1}^{t}$, then (4) becomes: $T_{i}^{t} \geq 1+F_{i-1}^{t}$. But since $t \in\left[r_{i}, d_{i}\right)$, we have $F_{i}^{t}=F_{i-1}^{t}+1$. Thus, we have:

$T_{i}^{t} \geq F_{i}^{t}-1+1=F_{i}^{t} \geq \min \left\{F_{i}^{t}, \frac{k}{2}-1\right\}$.

- If $\sigma_{i} \in L$ and $\sigma_{i} \notin R$. This means that $\sigma_{i}$ is accepted by $L R^{k}$ and $\sigma_{j}$ is rejected. By the on-line context, since the last revealed interval is $\sigma_{i}$, all the intervals which do not belong to $L$ have a release date equal to $r_{i}$ (otherwise they would belong to $L$ ). In particular, $\sigma_{j} \notin L$ because it is interrupted and thus it satisfies $r_{j}=r_{i}$. Moreover, by the manner the algorithm builds $L, \sigma_{i}$ has also a greater deadline than $\sigma_{j}$ (otherwise, $\sigma_{j} \in L$ and thus it would not be interrupted): $d_{j} \leq d_{i}$. We have 3 cases to consider:

- For all $t \notin\left[r_{i}, d_{i}\right)$, we have $F_{i}^{t}=F_{i-1}^{t}$ and $T_{i}^{t}=T_{i-1}^{t}$. Thus, by replacing $T_{i-1}^{t}$ by $T_{i}^{t}$ and $F_{i-1}^{t}$ by $F_{i}^{t}$ in the induction hypothesis, this particular case is checked.

- For all $t \in\left[r_{i}, d_{j}\right)$, we have $T_{i}^{t}=T_{i-1}^{t}$ because $\sigma_{j}$ is deleted but $\sigma_{i}$ is added. Since $\sigma_{i} \notin R$, there are at least $\left\lfloor\frac{k}{2}\right\rfloor$ intervals containing date $t$ having a deadline at least $d_{i}$. Thus, we have:

$T_{i}^{t} \geq\left\lfloor\frac{k}{2}\right\rfloor \geq \min \left\{F_{i}^{t}, \frac{k}{2}-1\right\}$

- For all $t \in\left[d_{j}, d_{i}\right)$, since $\sigma_{i}$ occupies a machine that was free at step $i-1$ of the algorithm, we have: $T_{i}^{t}=T_{i-1}^{t}+1$. By the induction hypothesis, we can rewrite this equation:

$$
T_{i}^{t} \geq 1+\min \left\{F_{i-1}^{t}, \frac{k}{2}-1\right\}
$$

If $\min \left\{F_{i-1}^{t}, \frac{k}{2}-1\right\}=\frac{k}{2}-1$, then (5) becomes:

$T_{i}^{t} \geq 1+\frac{k}{2}-1=\frac{k}{2}>\frac{k}{2}-1 \geq \min \left\{F_{i}^{t}, \frac{k}{2}-1\right\}$

If $\min \left\{F_{i-1}^{t}, \frac{k}{2}-1\right\}=F_{i-1}^{t}$, then (5) becomes: $T_{i}^{t} \geq 1+F_{i-1}^{t}$. But since $t \in\left[r_{i}, d_{i}\right)$, we have $F_{i}^{t}=F_{i-1}^{t}+1$. Thus, we have

$T_{i}^{t} \geq F_{i}^{t}-1+1=F_{i}^{t} \geq \min \left\{F_{i}^{t}, \frac{k}{2}-1\right\}$. We have checked the induction step and thus the lemma. 
Theorem 2 For proportional weights $\left(w_{i}=p_{i}\right), L R^{k}$ is $\frac{2}{1-\frac{2}{k}}$-competitive.

Proof. Let $O_{i}^{*}$ be the optimal (off-line) weight schedule of $\left\{\sigma_{1}, \ldots, \sigma_{i}\right\}$. Let $T_{i}^{* t}$ be the number of intervals of the schedule $O_{i}^{*}$ containing date $t$. Let $t$ be a date of the schedule $O$ returned by $L R^{k}$ on the input sequence $\sigma_{1}, \cdots, \sigma_{i}$ and $i$ be a step of the algorithm. If $\min \left\{F_{i}^{t}, \frac{k}{2}-1\right\}=F_{i}^{t}$ then by Lemma 3 , we have $T_{i}^{t} \geq F_{i}^{t} \geq T_{i}^{* t}$. Now, let us consider the case in which $\min \left\{F_{i}^{t}, \frac{k}{2}-1\right\}=\frac{k}{2}-1$. Since $O_{i}^{*}$ is valid, we have $T_{i}^{* t} \leq k$. Multiplying both sides by $\frac{1-\frac{2}{k}}{2}$, by remarking that $\frac{k}{2}\left(1-\frac{2}{k}\right)=\frac{k}{2}-1$ and by Lemma 3 , we obtain:

$\frac{T_{i}^{* t}}{2}\left(1-\frac{2}{k}\right) \leq \frac{k}{2}\left(1-\frac{2}{k}\right)=\frac{k}{2}-1 \leq T_{i}^{t}$. Thus, we have for all dates $t$ and for all steps $i$ : $\frac{2}{1-\frac{2}{k}} T_{i}^{t} \geq T_{i}^{* t}$. If we sum up this inequality for all dates $t$, we obtain that $L R^{k}$ is $\frac{2}{1-\frac{2}{k}}$-competitive.

Recall that $G O L^{r}$ is an optimal on-line algorithm for the SIZE and $L R^{k-r}$ is an on-line $\frac{2}{1-\frac{2}{k-r}}$-competitive algorithm for the PROPORTIONAL WEIGHTS problem. So, applying Theorem 1, we have:

Corollary 2 For all $1 \leq r \leq k-3, A B^{k}$ applied with $A^{r}=G O L^{r}$ and $B^{k-r}=L R^{k-r}$ is $\left(\frac{k}{r}, \frac{2 k}{\left(1-\frac{2}{k-r}\right)(k-r)}\right)$-competitive for the size and proportional weights criteria.

Note that the parameter $r$ that can be tuned in order to make $A B^{k}$ more precise for one of the objectives. For example, If we want $A B^{k}$ to get the same competitive ratio for the SIZE and for the PROPORTIONAL WEIGHT, we had to set $r=\frac{k-2}{3}$ and we obtain a couple of competitive ratios of $\left(\frac{3}{1-\frac{2}{k}}, \frac{3}{1-\frac{2}{k}}\right)$ which tends towards $(3,3)$ for large $k$.

\section{References}

1. E. Arkin And B. Silverberg, Scheduling jobs with fixed start and end times, Discrete Applied Mathematics, 18 (1987), pp. 1-8.

2. F. BAille, E. BAmpis, AND C. LAfOREST, A note on bicriteria schedules with optimal approximation ratios, Parallel Processing Letters, 14 (2004), pp. 315-323.

3. A. Bar-Noy, R. Canetti, S. Kutten, Y. Mansour, and B. Schieber, Bandwidth allocation with preemption, SIAM J. Comput., 28 (1999), pp. 1806-1828.

4. A. Borodin AND R. El-YANIV, Online computation and competitive analysis, Cambridge University press, 1998.

5. M. C. Carlisle AND E. L. Lloyd, On the $k$-coloring of intervals, Discrete Applied Mathemetics, 59 (1995), pp. 225-235.

6. U. Faigle AND M. NAWIJn, Note on scheduling intervals on-line, Discrete Applied Mathematics, 58 (1995), pp. 13-17.

7. A. Fiat And G. J. Woeginger, Online algorithms: The state of the art, LNCS no. 1442, Springer, 1998.

8. G. J. WoEgINGER, On-line scheduling of jobs with fixed start and end times, Theor. Comput. Sci., 130 (1994), pp. 5-16. 